Check for updates

Cite this: RSC Adv., 2019, 9, 23081

\title{
Surfactant-free synthesis of porous Au by a urea complex $\dagger$
}

\author{
Qiang Zhang, ${ }^{\mathrm{b}}$ Biao Zang ${ }^{\mathrm{b}}$ and Shaozhen Wang (D) *a
}

Received 11th June 2019

Accepted 7th July 2019

DOI: $10.1039 / c 9 r a 04372 a$

rsc.li/rsc-advances

\begin{abstract}
We report a facile surfactant-free synthetic method to obtain porous and hollow Au nanoparticles using only urea and $\mathrm{HAuCl}_{4} \cdot 4 \mathrm{H}_{2} \mathrm{O}$ as precursors at $200{ }^{\circ} \mathrm{C}$. The formation mechanism was investigated through X-ray diffraction, scanning electron microscopy, and high-resolution transmission electron microscopy. Moreover, the prepared Au nanocrystals present superior catalytic performance for the reduction of 4-nitrophenol in comparison with solid Au nanoparticles. The catalytic efficiency of porous and hollow Au was nearly 6 times higher than that of Au nanoparticles. Furthermore, the porous and hollow Au maintained excellent stability even after 10 catalytic cycles. Therefore, the as-synthesized porous Au nanoparticles will have potential applications in organic catalysis, biosensing, drug delivery, water pollutant removal, and so on.
\end{abstract}

\section{Introduction}

Noble metal nanoparticles still attract considerable attention due to their chemical stability, versatility, and considerable potential applications in sensing, ${ }^{1}$ medical application, ${ }^{2-4}$ catalysis, ${ }^{5-7}$ etc. To improve their application, noble metal nanostructures with diverse shapes have been synthesized, which include nanorods,${ }^{8,9}$ nanocages, ${ }^{10}$ nanostars, ${ }^{11}$ and porous structures. ${ }^{12,13}$ Among these structures, porous nanoparticles are particularly attractive because of their larger internal cavity and specific surface area. ${ }^{14}$

Various chemical or physical synthesis methods have been developed for preparing metal nanocrystals with a porous feature, including chemical etching, ${ }^{15}$ the template method, ${ }^{16,17}$ and seed-mediated growth. ${ }^{18}$ As a typical example, Wang et al. prepared porous Pd nanoparticles by a seed-mediated method in an aqueous solution using cetyltrimethylammonium chloride (CTAC) as a stabilizing agent. ${ }^{18}$ Surfactants, such as poly(vinylpyrrolidone) (PVP) or cetyltrimethylammonium bromide (CTAB), are key for the formation of porous structures in most of the reported syntheses. ${ }^{19}$ However, the existence of surfactants on the surface of the nanoparticles becomes a huge obstacle in decreasing the active sites for the catalytic reaction..$^{20,21}$ Moreover, the removal of surfactants is not only a tedious process but also can lead to an agglomeration of particles, which will also decrease their catalytic activity. The

${ }^{a}$ AnHui Provincial Engineering Research Center for Polysaccharide Drugs, Institute of Synthesis and Application of Medical Materials, Department of Pharmacy, Wannan Medical College, Wuhu 241002, P. R. China.E-mail:wangshaozhen@wnmc.edu.cn ${ }^{b}$ AnHui Key Laboratory of Chemo/Biosensing, College of Chemistry and Materials Science, AnHui Normal University, WuHu, 241000, P. R. China

$\dagger$ Electronic supplementary information (ESI) available. See DOI: 10.1039/c9ra04372a surfactant-free synthetic strategy, which will not introduce the stabilizer during the growth procedure, is thus highly attractive. Up to now, there are some studies on the surfactant-free synthesis of solid Au nanoparticles. ${ }^{22-24}$ For example, Lai et al. prepared homogeneous Au nanoparticles encapsulated by polythiophene analogues. ${ }^{25}$ However, the growth of the porous and hollow $\mathrm{Au}$ nanoparticles is still rarely reported..$^{26}$ Such an architecture will undoubtedly increase the catalytic sites and thus enhance the catalytic performances.

Herein, we report the surfactant-free synthesis of porous and hollow Au (PHAuNPs) via a incinerate precursors, namely, Gold-Urea Complex (GUC) precursors. These complexes were formed by mixing urea with $\mathrm{HAuCl}_{4} \cdot 4 \mathrm{H}_{2} \mathrm{O}$. The precursors were reduced and grown into PHAuNPs under calcination at $200^{\circ} \mathrm{C}$. We found that PHAuNPs show spherical morphology with a size of $50 \mathrm{~nm}$. It was noticed that the nanostructure shows a hollow feature formed by the nanoparticles with a gap of $1 \mathrm{~nm}$ externally. The component nanoparticles had a size of around $5 \mathrm{~nm}$. Furthermore, the catalytic performance of the PHAuNPs was assessed by employing the reduction of 4-nitrophenol (4-NP) to 4-aminophenol (4-AP) as a model reaction. The results revealed that the catalytic activity of PHAuNPs was 6 times higher than that of the commercial solid gold nanoparticles. Moreover, the PHAuNPs also exhibited highly improved stability during the catalytic examinations.

\section{Experimental}

\subsection{Synthesis of Gold-Urea Complex (GUC) precursors}

In our proposed synthesis, $\mathrm{HAuCl}_{4}(5.89 \mathrm{mM}, 1.0 \mathrm{~mL})$ was stirred with urea $(0.2 \mathrm{~g}, 10 \mathrm{~mL})$ at room temperature for $5 \mathrm{~min}$. Then, the mixture was transferred into a round-bottom flask with a reflux condenser to maintain the fast stirring at $100{ }^{\circ} \mathrm{C}$ 
for $10 \mathrm{~min}$. The precipitate obtained was washed with deionized water and ethanol three times and collected for further characterization.

\subsection{Synthesis of porous and hollow Au nanoparticles (PHAuNPs) and porous and solid Au nanoparticles (PSAuNPs)}

Briefly, GUC was diluted with $1 \mathrm{~mL}$ ethanol and converted to a neat ampulla via ultrasonication for $2 \mathrm{~min}$. The ampulla was then incinerated at $160{ }^{\circ} \mathrm{C}$ or $200{ }^{\circ} \mathrm{C}$. The products obtained were subsequently washed with deionized water and ethanol three times and preserved for further application.

\subsection{Catalytic reduction of 4-NP}

Initially, $5 \mu \mathrm{L}$ of 4 -NP ( $60 \mathrm{mM}$ ) diluted with $2.5 \mathrm{~mL}$ of deionized water was added into the standard quartz cuvettes at room temperature, followed by the rapid addition of $150 \mu \mathrm{L}$ of freshly prepared ice-cold $\mathrm{NaBH}_{4}$ solution $(1.0 \mathrm{M})$. Then, the PHAuNPs $\left(0.5 \mathrm{mg} \mathrm{mL}{ }^{-1}, 100 \mu \mathrm{L}\right)$ and the commercial gold nanoparticles $\left(0.5 \mathrm{mg} \mathrm{mL}{ }^{-1}, 100 \mu \mathrm{L}\right)$ were added into cuvettes sequentially to compare the catalytic activity. The reaction process was monitored by measuring the UV-vis absorption spectra with a time interval of $80 \mathrm{~s}$. The stabilities of the catalysts were tested by a ten-run recycling experiment. After each reaction, $5 \mu \mathrm{L}$ of $4-\mathrm{NP}$ (60 $\mathrm{mM})$ was directly added into the reaction mixture for the second to tenth runs.

\subsection{Chemicals and materials}

$\mathrm{HAuCl}_{4} \cdot 3 \mathrm{H}_{2} \mathrm{O}$, sodium borohydride $\left(\mathrm{NaBH}_{4}\right)$, and 4-nitrophenol (4-NP) were obtained from Aldrich. Urea and ethanol were purchased from Shantou Xilong and Fuchen TianJin, respectively. The commercial solid gold nanoparticles were purchased from XFNANO (Size: $50 \mathrm{~nm}$, Sodium citrate as a reducing agent). All the aqueous solutions were prepared with twice-distilled water, obtained from a water purifying machine (Hokee, $18.25 \mathrm{M} \Omega \mathrm{cm}^{-1}$ ).

\subsection{Characterization}

SEM images were obtained using a scanning electron microscope (SEM, Hitachi S-4800 Japan). TEM images were captured on a transmission electron microscope (Tecnai G20 S-Twin, Holland). The UV-vis absorbance of 4-NP was monitored by a lambda 35 spectrophotometer. FT-IR spectra were obtained on a Hitachi FTIR-8400S using $\mathrm{KBr}$ pellets. Elemental Analyzer (Vario EL cube, Germany) was used to detect the elements (C, H, and $\mathrm{N}$ ). The surface elemental composition was obtained by Xray photoelectron spectroscopy (XPS, Thermo Fisher Scientific, UK) using Al Ka X-ray radiation (1486.8 eV) for excitation. X-ray diffraction (XRD) patterns were collected on a Germany Bruker D8 ADVANCE diffractometer equipped with $\mathrm{Cu} \mathrm{K} \alpha$ radiation ( $\lambda$ $=1.5418 \AA$ ). Au was determined by an inductive coupled plasma atomic emission spectrometer (ICP, Atomscan Advantage, Thermo Jarrell Ash Corporation).

\section{Results and discussion}

We obtained porous Au nanoparticles with different detailed structures under different temperatures. The products were characterized by transmission electron microscopy (TEM). Interestingly, the Au nanocrystals show a porous structure with a solid center when it was obtained at a low temperature of $160{ }^{\circ} \mathrm{C}$ (Fig. 1a). However, the morphology changes into a hollow architecture when the growth temperature was increased to a high temperature of $200{ }^{\circ} \mathrm{C}$ (Fig. 1b). It should be noted that the $\mathrm{Au}$ nanostructures still possess a porous feature when grown in this condition. Furthermore, the magnified TEM images clearly show that both types of $\mathrm{Au}$ nanoparticles are composed of particles of $5 \mathrm{~nm}$.

The absorption spectra of the aqueous solutions of $\mathrm{HAuCl}_{4}$, GUC, and PHAuNPs were monitored at $292 \mathrm{~nm}, 260 \mathrm{~nm}$, and $575 \mathrm{~nm}$, respectively (Fig. $\mathrm{S} 1 \dagger$ ). We can find a blue shift in the absorption of GUC compared with that of $\mathrm{HAuCl}_{4}$, which may be caused by the formation of a complex between $\mathrm{HAuCl}_{4}$ and urea. Furthermore, inductive coupled plasma atomic emission spectrometer and elemental analyzer were used to analyze the element percentage of the sample (Table 1), from which the chemical formula of GUC was determined to be $\mathrm{Au}\left(\mathrm{N}_{2} \mathrm{H}_{4} \mathrm{CO}\right)$. $\mathrm{H}_{2} \mathrm{O}$. Furthermore, the infrared spectra of GUC(I), urea(II), and PHAuNPs (III) were obtained (Fig. S2 $\dagger$ ). The spectra reveal that after an interaction between urea and gold chloride, the absorption peak in the spectrum of GUC shows a little shift from $1681 \mathrm{~cm}^{-1}$ to $1629 \mathrm{~cm}^{-1}$ compared to that in the spectrum of urea. Moreover, a characteristic band of terminal isocyanate at $2171 \mathrm{~cm}^{-1}$ and of a coordinated amide group at 3490$3400 \mathrm{~cm}^{-1}$ was detected. Additionally, the band at $574 \mathrm{~cm}^{-1}$ was attributed to $\nu(\mathrm{Au}-\mathrm{N})$. However, the spectrum of PHAuNPs shows no characteristic bands of coordinated urea because of the high-temperature calcination.

The crystal structure of the products obtained from different calcination times was investigated by powder X-ray diffraction (XRD). As shown in Fig. 2, the lower blue line is the JCPDS data of the standard Au (card number 65-8601). The GUC (Fig. 2b, I) has no characteristic diffraction peak, indicating that $\mathrm{Au}$ nanocrystals were not formed. After GUC was calcined at $200{ }^{\circ} \mathrm{C}$ for about $20 \mathrm{~min}$, the products turn brownish gray (Fig. 2a, II) and the characteristic peaks of Au appear in the spectra (Fig. 2b, II). Further, the characteristic peaks of gold in the purple-red products (Fig. 2a, III) become sharp as the calcination time

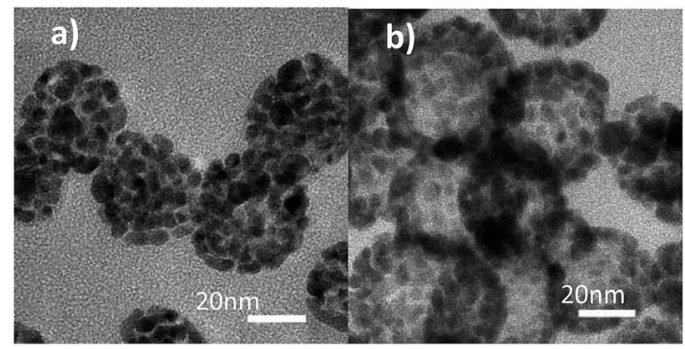

Fig. 1 TEM image of the PSAuNPs obtained at $160{ }^{\circ} \mathrm{C}(\mathrm{a})$; and PHAuNPs obtained at $200^{\circ} \mathrm{C}$ (b). 
Table 1 The results of analysis of each component

\begin{tabular}{lllll}
\hline Element & $\mathrm{Au}$ & $\mathrm{N}$ & $\mathrm{C}$ & $\mathrm{H}$ \\
\hline Actual value/wt\% & 69.67 & 10.08 & 3.61 & 2.2 \\
Theory value/wt\% & 71.63 & 10.18 & 4.36 & 2.18
\end{tabular}

a)

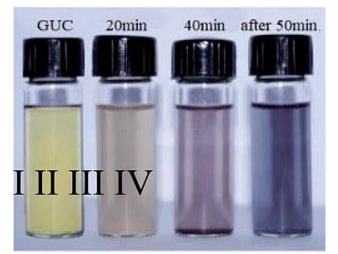

c)

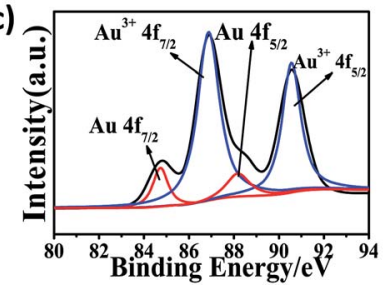

b)

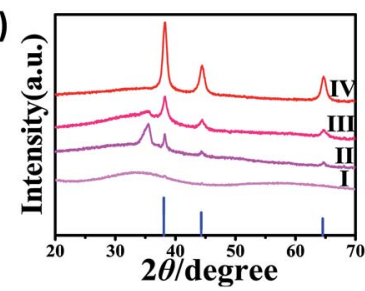

d)

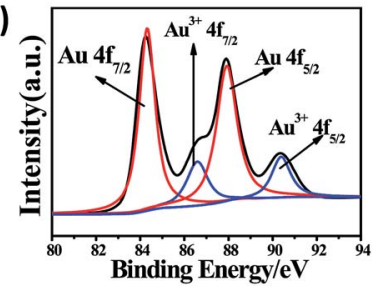

Fig. 2 (a) The color change with time gradient calcination. (b) XRD patterns of products under different calcination time (I) GUC (origin), (II) $20 \mathrm{~min}$, (III) $40 \mathrm{~min}$, (IV) $60 \mathrm{~min}$. XPS spectra of Au $4 \mathrm{f}$ of the asprepared GUC (c) and PHAuNPs (d) deposited on the silica wafer.

was increased to $40 \mathrm{~min}$ (Fig. 2b, III). Moreover, the intensity of the peak observed at $35.49^{\circ}$ decreased. Further, the XRD characteristic peaks of the black products (Fig. 2a, IV), obtained after calcination for 50 min (Fig. 2b, IV) were consistent with the standard diffraction peaks of $\mathrm{Au}$ at $38.19^{\circ}, 44.43^{\circ}$ and $64.69^{\circ}$, which correspond to (111), (200), (220), respectively. Therefore, this proved that the GUC was reduced to gold through a hightemperature reaction.

X-ray photoelectron spectroscopy (XPS) was further employed to characterize the chemical state of the GUC and PHAuNPs. The Au $4 \mathrm{f}$ XPS spectrum of the GUC displays two strong peaks at 86.89 and $90.56 \mathrm{eV}$ (Fig. 2c), which was assigned to $\mathrm{Au}^{3+} 4 \mathrm{f}_{7 / 2}$ and $\mathrm{Au}^{3+} 4 \mathrm{f}_{5 / 2}$, respectively. This result indicates that $\mathrm{Au}^{3+}$ is the dominant species at this stage. Further, the $\mathrm{N} 1 \mathrm{~s}$ peaks observed at $398.88 \mathrm{eV}$ (Fig. S5a $\dagger$ ) was assigned to $\mathrm{C}-\mathrm{N}$ bonds. The $\mathrm{C} 1 \mathrm{~s}$ at $284.83 \mathrm{eV}, 285.98 \mathrm{eV}$, and $288.18 \mathrm{eV}$ (Fig. S5b $\dagger$ ) correspond to $\mathrm{C}-\mathrm{C}$ bonds, $\mathrm{C}-\mathrm{O}$ bonds, and $\mathrm{C}=\mathrm{O}$ bonds, respectively. ${ }^{27}$ The survey spectrum again confirms that the GUC was composed of Au, N, C, and O. Thus, these results establish the metal-urea complex structure of GUC. After calcination at $200^{\circ} \mathrm{C}$, the XPS spectrum of the obtained product show two peaks at 84.32 and $87.77 \mathrm{eV}$, undoubtedly suggesting the $\mathrm{Au}^{0}$ chemical state (Fig. 2d). The above survey result demonstrates that the reactive metal ions were reduced from their positively charged state during the thermal annealing process.

We also investigated the growth process in this study. Initially, GUC was slightly yellow in color (Fig. S3a †). The corresponding samples exhibited a spherical morphology and a good size dispersion of $\sim 50 \mathrm{~nm}$ diameter, as characterized at low magnification under SEM imaging (Fig. S3b and $\mathrm{c} \dagger$ ). A single nanoparticle of GUC was focused and amplified under a transmission electron microscope, where it was observed that the GUC changed from a solid spherical structure to a porous and hollow structure in a short time, which was due to the strong electron beams (Fig. S3e†).

As mentioned above, the GUC changed into porous $\mathrm{Au}$ nanoparticles by the strong electron beams under the high magnified TEM. Hence, we captured the beam irradiation timedependent TEM images. Fig. S4a $\dagger$ shows the original low magnification TEM image of GUC (fast-focus shooting). The GUC dispersed homogeneously, with the increase in time. In addition, an increasingly large number of cavities were observed inside the GUC. Meanwhile, the solid parts also began to produce porous structures, and the nanoparticles started to fuse and attach to each other (Fig. S4b to d $\dagger$ ). This indicates that the complexes will be reduced to PHAuNPs by giving high energy gradually. Hence, the GUC was calcined to obtain PHAuNPs. After the evaporation of GUC/ethanol dispersion, the color of the products changed from yellow to brownish gray, then turned purple-red, and finally turned black (Fig. 2a).

We further explored the mechanism of the reaction, which reveals that the GUC shape changes on heating under different conditions. The amorphous GUC shows a good dispersion of $50 \mathrm{~nm}$ (Fig. 3b). After incinerating at $160{ }^{\circ} \mathrm{C}$, the PSAuNPs were formed with sizes of around $5 \mathrm{~nm}$ and a gap of $1 \mathrm{~nm}$ both internally and externally (Fig. 3d). When the PHAuNPs were obtained on calcination at $200{ }^{\circ} \mathrm{C}$ for about 30 to $50 \mathrm{~min}$ (Fig. 3f), the nanostructure maintained the original size of $50 \mathrm{~nm}$, while the nanoparticles of $5 \mathrm{~nm}$ size were arranged with a gap of $1 \mathrm{~nm}$ externally to form the hollow structure. We speculate that the reason for the temperature-dependent morphological change was the rapid thermal decomposition of urea to $\mathrm{NH}_{3}$ and $\mathrm{CO}_{2}$ at $200{ }^{\circ} \mathrm{C}$. First, the shell of the PHAuNPs was formed due to the fast heating. Urea and ethanol acted together as weak reducing agents, and the gas generated by urea pyrolysis because of the surface tension induces gold particles to move to the sphere surface to form the porous hollow structure. However, urea cannot pyrolyze rapidly at $160{ }^{\circ} \mathrm{C}$. The whole particle was heated uniformly and decomposed and reduced slowly, and the internal gas could not be released quickly to induce the assembly of reduced gold particles to move to the sphere surface. As a result, the PSAuNPs were formed. Both PHAuNPs and PSAuNPs will eventually fusion and deform when heated after $50 \mathrm{~min}$. The small particles overlapped and fused with each other. Finally, larger particles with a diameter of about $20 \mathrm{~nm}$ were formed embedded in the polyhedron (Fig. $3 \mathrm{~h}$ ).

The catalytic properties of PHAuNPs were evaluated via the reduction reaction of 4-NP to 4-AP in the presence of excess $\mathrm{NaBH}_{4}$, which was monitored by collecting ultraviolet-visible absorption (UV-vis) spectra. ${ }^{28}$ When $\mathrm{NaBH}_{4}$ was added to 4$\mathrm{NP}$, a yellow solution was obtained, and the time-dependent absorption spectra were measured immediately. The spectra show a maximum absorption peak at $400 \mathrm{~nm}$, which was the absorption peak of 4-nitrophenolate. Further, the yellow 


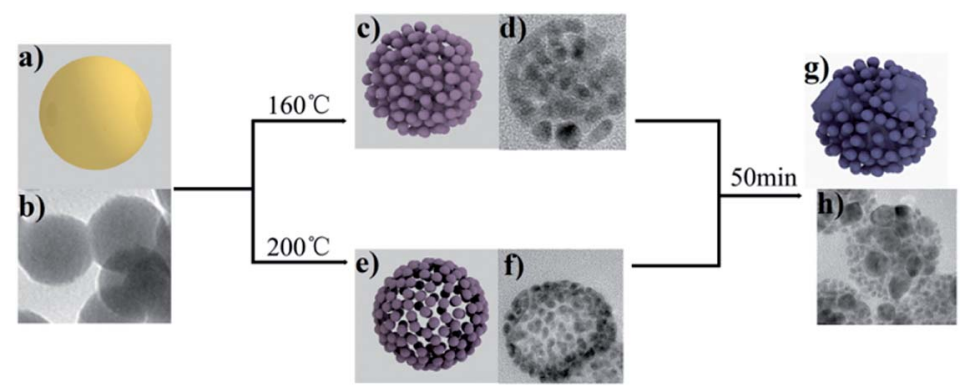

Fig. 3 (a) The schematic diagram and (b) TEM image of GUC; (c) the schematic diagram and (d) TEM image of PSAuNPs obtained at $160{ }^{\circ} \mathrm{C}$; (e) the schematic diagram and (f) TEM image of PHAuNPs obtained at $200{ }^{\circ} \mathrm{C}$; (g) the schematic diagram and (h) TEM images of Au calcined after $50 \mathrm{~min}$.

solution without catalysts was placed indoors for 18 hours before characterizing it using a UV-vis spectrophotometer. The red line showed no significant change (Fig. S5 $\dagger$ ), indicating that 4-nitrophenol reduced slowly. After the addition of PHAuNPs, the absorption peak at $400 \mathrm{~nm}$ decreases rapidly, and a new absorption peak appeared at $300 \mathrm{~nm}$. This was mainly due to the catalytic reduction of 4-NP to 4-AP (Fig. 4a). As the reaction proceeded, the maximum absorption peak $(400 \mathrm{~nm})$ of $4-\mathrm{NP}$ decreased rapidly and disappeared completely after the reaction was carried out for just $240 \mathrm{~s}$. Since the amount of $\mathrm{NaBH}_{4}$ was in excess, this reduction reaction was assumed to follow pseudo first-order kinetics, and the rate constant was thus utilized to evaluate the reaction rate. Fig. $4 \mathrm{~b}$ shows the linear fit between the reaction time and $-\ln \left(C_{t} / C_{0}\right)$ for the catalytic reduction of 4NP using PHAuNPs as the catalyst. The kinetic rate constant $K_{\text {app }}$, calculated directly from the slope, was estimated to be 12.1 $\times 10^{-3} \mathrm{~s}^{-1}$.

a)
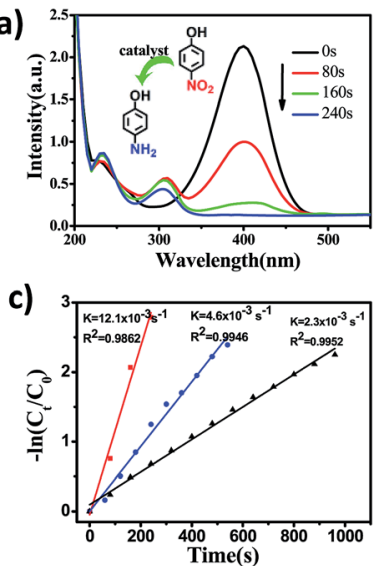

Fig. 4 (a) Time-dependent absorption spectra of the reduction of 4NP to 4-AP catalyzed by PHAuNPs. (b) The black and white squares stand for the $C_{t} / C_{0}$ and $-\ln \left(C_{t} / C_{0}\right)$ versus reaction time, respectively, for the reduction of $4-\mathrm{NP}$ by $\mathrm{NaBH}_{4}$ over PHAuNPs. $\mathrm{C}_{0}$ corresponds to the initial intensity of the absorption at $400 \mathrm{~nm}$, and $C_{t}$ is the absorption peak at different reaction time $(t)$. (c) $-\ln \left(C_{t} / C_{0}\right)$ versus reaction time for the reduction of 4-NP for PHAuNPs (red line), PSAuNPs (bule line) and commercial solid gold nanoparticles (black line), (d) $K_{\text {app }}$ values under different catalytic cycles in the reduction of 4-NP to 4-AP in the presence of PHAuNPs.
In order to prove the enhancement in the catalytic activity of PHAuNPs, PSAuNPs and the commercial solid gold nanoparticles with the same size were used as catalysts for a comparison. The catalytic reduction of 4-NP to 4-AP was also used to discuss the catalytic activity of the PSAuNPs and commercial solid gold nanoparticles (Fig. S7 and S8†). Compared to PHAuNPs, the catalytic rates of the PSAuNPs and commercial solid gold nanoparticles were lower, with the $K_{\text {app }}$ being $4.6 \times 10^{-3} \mathrm{~s}^{-1}$ and $2.3 \times 10^{-3} \mathrm{~s}^{-1}$, respectively (Fig. 4c). In other words, the as-prepared PHAuNPs show an accelerated reaction rate when they were employed as the catalysts, which was nearly 2.6 and 6 times that of PSAuNPs and commercial gold catalysts, respectively. The excellent catalytic performance of PHAuNPs was attributed to the porous and hollow structure. First, the porous cavity composed of about $5 \mathrm{~nm}$ gold nanoparticles endows the PHAuNPs with a larger specific surface area, which in turn provides more reactive sites for the same size. Second, the hollow structure was more favorable for the exchange of the substrate on the catalyst than the porous and solid structure. Furthermore, the surface of the samples was not covered by the surfactant. The PHAuNPs can thus provide more effective reactive sites. As we all know, recyclability is also very important for nanocatalysts in practical applications. ${ }^{29}$ The catalytic reduction product of 4-AP will adsorb on the surface of catalysts, which can lead to catalyst poisoning. ${ }^{30}$ We thus also investigated the recyclability of the PHAuNPs catalysts. Fig. 4d displays the values of $K_{\text {app }}$ for the successive reactions. It was found that the catalytic activity of the PHAuNPs slightly increased after the first run. Presumably, the catalyst was activated by $\mathrm{NaBH}_{4}$. The $K_{\text {app }}$ after ten cyclic measurements for the reduction of 4-NP could still reach $11.7 \times 10^{-3} \mathrm{~s}^{-1}$, and exhibits almost no change compared with the initial cycles.

\section{Conclusions}

In summary, we have synthesized PHAuNPs using a surfactantfree method. The obtained Au nanocrystals show a novel porous and hollow structure, which provides high active sites for the catalytic reaction. Moreover, the surface of the PHAuNPs was clean, which is also beneficial for improving the catalytic activity. The catalytic performance of the as-prepared porous PHAuNPs toward the reduction of 4-NP to 4-AP revealed a 6-fold 
higher $K_{\text {app }}$ than that of the commercial gold nanoparticles. The stability was also found to be highly improved through cycling tests. Therefore, this study provides a new way to construct clean noble metal nanoparticles with special structures and properties in the future.

\section{Conflicts of interest}

There are no conflicts to declare.

\section{Acknowledgements}

This study was financially supported by the National Science Foundation of China (21301008, 21175002), Anhui Science and Technology Major Project (17030801021), Open Foundation of CAS Key Laboratory of Analytical Chemistry for Living Biosytems, ACL, Open Foundation of The Key Laboratory of Functional Molecular Solids, Ministry of Education (14007) and the members of Institute of Synthesis and Application of Medical Materials.

\section{References}

1 W. He, X. Wu, J. Liu, X. Hu, K. Zhang, S. Hou, W. Zhou and S. Xie, Chem. Mater., 2010, 22, 2988-2994.

2 I. Fratoddi, I. Venditti, C. Cametti and M. V. Russo, J. Mater. Chem. B, 2014, 2, 4204-4220.

3 H. Cai and P. Yao, Nanoscale, 2013, 5, 2892-2900.

4 Y. Niidome, A. T. Haine and T. Niidome, Chem. Lett., 2016, 45, 488-498.

5 K. Zhou and Y. Li, Angew. Chem., Int. Ed. Engl., 2012, 51, 602613.

6 W. Si, J. Li, H. Li, S. Li, J. Yin, H. Xu, X. Guo, T. Zhang and Y. Song, Nano Res., 2013, 6, 720-725.

7 W. Yang, Q. Zhang and C. Peng, RSC Adv., 2019, 9, 931-939. 8 X. Huang, S. Neretina and M. A. El-Sayed, Adv. Mater., 2009, 21, 4880-4910.

9 H. Chen, F. Yuan, S. Wang, J. Xu, Y. Zhang and L. Wang, Biosens. Bioelectron., 2013, 48, 19-25.

10 J. Chen, C. Glaus, R. Laforest, Q. Zhang, M. Yang, M. Gidding, M. J. Welch and Y. Xia, Small, 2010, 6, 811-817.

11 H. Yuan, A. M. Fales and T. Vo-Dinh, J. Am. Chem. Soc., 2012, 134, 11358-11361.
12 G. W. Nyce, J. R. Hayes, A. V. Hamza, H. Joe and J. H. Satcher Jr, Chem. Mater., 2007, 19, 344-346.

13 Y. Qin, Y. Lu, W. Pan, D. Yu and J. Zhou, RSC Adv., 2019, 9, 10314-10319.

14 Z. Qi, U. Vainio, A. Kornowski, M. Ritter, H. Weller, H. Jin and J. Weissmüller, Adv. Funct. Mater., 2015, 25, 2530-2536.

15 H. Wu, P. Wang, H. He and Y. Jin, Nano Res., 2012, 5, 135144.

16 S. Pedireddy, H. K. Lee, W. W. Tjiu, I. Y. Phang, H. R. Tan, S. Q. Chua, C. Troadec and X. Y. Ling, Nat. Commun., 2014, 5, 4947.

17 R. Kumar, A. N. Maitra, P. K. Patanjali and P. Sharma, Biomaterials, 2005, 26, 6743-6753.

18 F. Wang, C. Li, L. D. Sun, C. H. Xu, J. Wang, J. C. Yu and C. H. Yan, Angew. Chem., Int. Ed. Engl., 2012, 51, 4872-4876.

19 M. Chen, B. Wu, J. Yang and N. Zheng, Adv. Mater., 2012, 24, 862-879.

20 S. Wang, L. Kuai, Y. Huang, X. Yu, Y. Liu, W. Li, L. Chen and B. Geng, Chem.-Eur. J., 2013, 19, 240-248.

21 Q. Cao, K. Yuan, J. Yu, J. J. Delaunay and R. Che, J. Colloid Interface Sci., 2017, 490, 23-28.

22 T. T. Guo, G. Yu, Y. Zhang, H. Y. Xiang, F. F. Chang and C. J. Zhong, J. Phys. Chem. C, 2017, 121, 3108-3116.

23 A. T. Miah, S. K. Bharadwaj and P. Saikia, Powder Technol., 2017, 315, 147-156.

24 M. A. Wall, S. Harmsen, S. Pal, L. Zhang, G. Arianna, J. R. Lombardi, C. M. Drain and M. F. Kircher, Adv. Mater., 2017, 29, 1605622.

25 C. H. Lai, I. C. Wu, C. C. Kang, J. F. Lee, M. L. Ho and P. T. Chou, Chem. Commun., 2009, 15, 1996-1998.

26 M. Z. Guo, J. He, Y. Li, S. Ma and X. H. Sun, J. Hazard. Mater., 2016, 310, 89-97.

27 W. Wen, J. Yao, Y. Luo, L. Lai and J. Wu, Ceram. Int., 2018, 44, 4381-4384.

28 K. Gu, X. Pan, W. Wang, J. Ma, Y. Sun, H. Yang, H. Shen, Z. Huang and H. Liu, Small, 2018, 14, 1801812.

29 X. Lu, X. Bian, G. Nie, C. Zhang, C. Wang and Y. Wei, J. Mater. Chem., 2012, 22, 12723.

30 T. Yu, J. Zeng, B. Lim and Y. Xia, Adv. Mater., 2010, 22, 51885192. 